\title{
Independent Measurement of Oxygen Consumption and Oxygen Delivery
}

\author{
Robert E. Cilley, M.D., Theodore Z. Polley, JR., M.D., Joseph B. Zwischenberger, M.D., \\ John M. Toomasian, C.C.P., AND Robert H. BaRTletT, M.D., F.C.C.P.
}

University of Michigan Medical Center, Department of Surgery, Ann Arbor, Michigan 48109-0331

Submitted for publication January 11, 1988

This experiment investigated the relationship between oxygen consumption and oxygen delivery (cardiac output $X$ arterial oxygen content) in an acute canine model. Previous studies investigating this relationship have largely utilized methods in which oxygen consumption and oxygen delivery were each calculated using the same measured variables resulting in mathematical coupling of variables. In this study, oxygen consumption was measured directly by closed-circuit spirometry. Oxygen delivery was independently controlled using right atrial bypass. These techniques assured that the values for oxygen consumption and oxygen delivery were derived entirely independently. In our study using 11 dogs, we found that oxygen consumption was constant and independent of oxygen delivery at oxygen delivery levels $>8.0 \mathrm{cc} / \mathrm{kg} / \mathrm{min}$ (normal canine oxygen delivery $20-25$ $\mathrm{cc} / \mathrm{kg} / \mathrm{min}$ ). Oxygen consumption was linearly related to oxygen delivery at oxygen delivery levels $\leqslant 8.0 \mathrm{cc} / \mathrm{kg} /$ min. Below this critical level of oxygen delivery, animals were more acidotic and hypotensive than at higher delivery levels. (c) $198 y$ Academic Press, Inc.

\section{INTRODUCTION}

The precise relationship between oxygen delivery $\left(\dot{D}_{2}\right)$ and oxygen consumption $\left(\dot{V O}_{2}\right)$ has yet to be defined completely for all normal and pathologic conditions. Under normal physiologic conditions in mammals, oxygen delivery exceeds tissue oxygen needs by a factor of four or five to one. This relationship is commonly expressed as either the coefficient of oxygen delivery $\left(\mathrm{COD}=\dot{D} \mathrm{O}_{2} /\right.$ $\dot{V} \mathrm{O}_{2}$ ) or its reciprocal, the oxygen extraction ratio (ER $=\dot{V} \mathrm{O}_{2} / \dot{D O}_{2}$ ). Oxygen delivery can increase considerably by increasing cardiac output in response to large increases in oxygen consumption associated with exercise. Under conditions of reduced oxygen delivery (including hypoxia, anemia, and low cardiac output states) tissue oxygen needs may barely be met. If oxygen delivery is lowered sufficiently, compensatory mechanisms will be exhausted and low oxygen delivery will eventually limit oxygen consumption. This level of oxygen delivery is referred to as the anaerobic threshold, below which lactic acidosis occurs and the organism deteriorates.

Recent clinical reports have shown that under certain pathologic conditions, oxygen consumption may vary directly with oxygen delivery at high levels of oxygen delivery $[1,2]$. Data were presented to show that oxygen consumption increased as oxygen delivery was increased in patients with the adult respiratory distress syndrome, although the mechanism by which this could occur is not clear. Animal studies that investigated the relationship between oxygen consumption and oxygen delivery have shown supply dependent oxygen consumption at very low levels of oxygen delivery induced by anemia, hypoxia, or low cardiac output [3-6].

In previous reports, the methods used to calculate oxygen delivery and consumption often utilized the same measured variables, making the reported relationships between delivery and consumption difficult to interpret. Either oxygen consumption was measured directly and oxygen delivery was calculated from that oxygen consumption, or cardiac output was measured and oxygen consumption and oxygen delivery were both calculated from this value.

The management of critically ill surgical patients includes manipulations that affect both oxygen delivery and oxygen consumption. A clearer understanding of the relationship between oxygen consumption and delivery during different disease states is necessary. Both clinical and laboratory studies using more precise methods are needed.

This report presents the results of experiments designed to investigate further the relationship between oxygen consumption and oxygen delivery. A canine model was used in which oxygen consumption was measured directly and independently of oxygen delivery, and oxygen delivery was controlled.

\section{METHODS}

Experimental design. Eleven mongrel dogs weighing 15-20 kg were used. After an overnight fast, pentobarbital anesthesia $(30 \mathrm{mg} / \mathrm{kg})$ was administered. The animals 


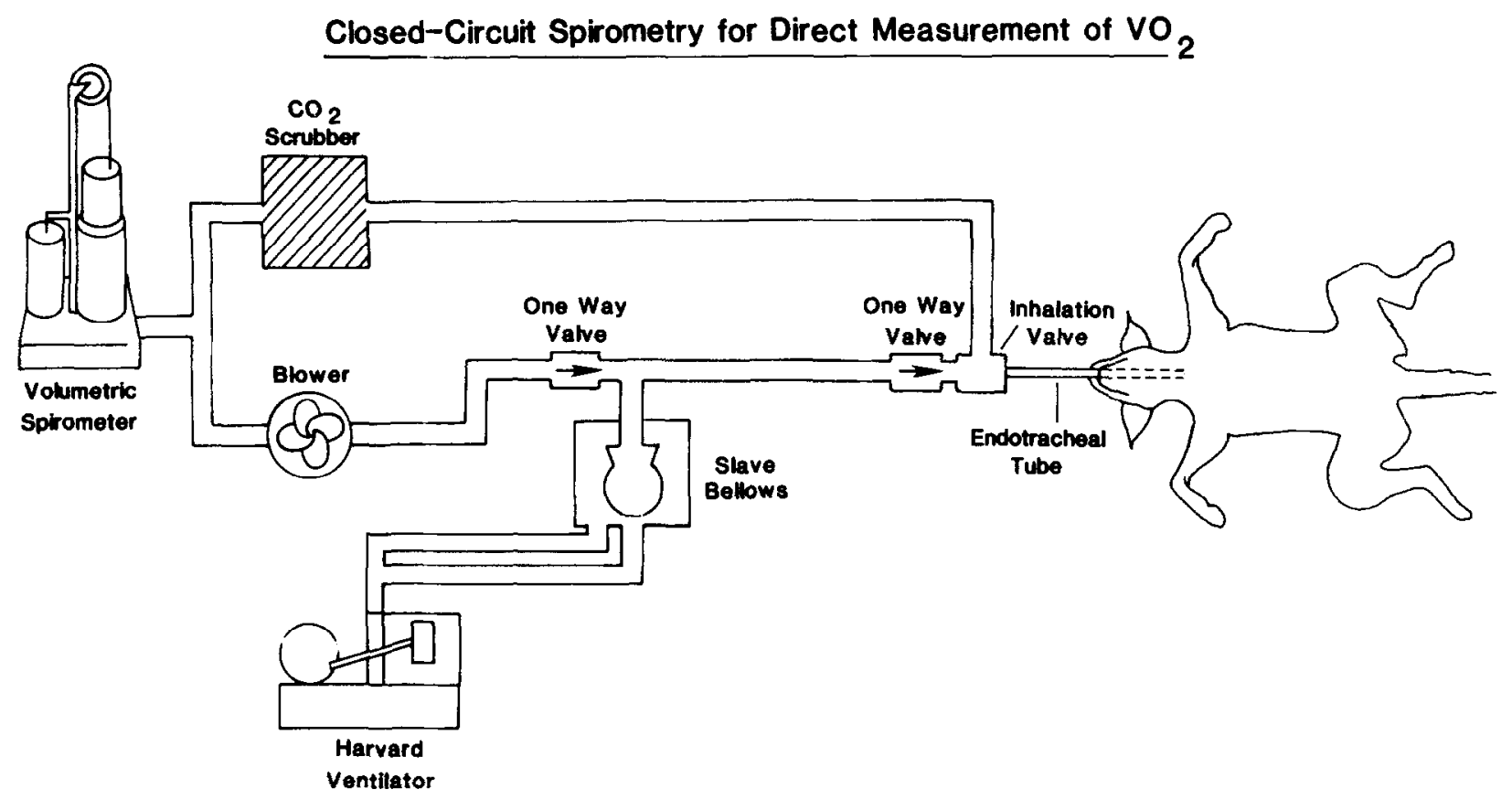

FIG. 1. Schematic diagram of closed-circuit spirometry system used for measuring oxygen consumption.

were intubated with a cuffed endotracheal tube and ventilated to a $p \mathrm{CO}_{2}$ of $35-45 \mathrm{~mm} \mathrm{Hg}$. Five percent dextrose and lactated Ringer's solution was administered at $5 \mathrm{cc} /$ $\mathrm{kg} / \mathrm{hr}$ throughout the experiment by a jugular venous catheter. Pancuronium bromide ( $3 \mathrm{mg}$ ) was given for paralysis. Additional pentobarbital and pancuronium were administered as needed. An eight-French femoral arterial catheter was placed for blood pressure monitoring and sampling. The animal was then ventilated with the closedcircuit spirometry system (see below under "oxygen consumption measurement"). Sequential oxygen consumption $\left(\dot{V} \mathrm{O}_{2}\right)$ measurements were begun. Right atrial bypass and pulmonary bypass were instituted and oxygen consumption was directly measured at different levels of oxygen delivery. Oxygen delivery was varied over a wide range in each dog, by changing cardiac output through alterations of the right atrial bypass pump speed and/or by selectively shunting venous blood to the left atrium. The hemoglobin level in a particular dog was dependent upon the priming solution in the bypass circuit. Heterologous canine blood was used in some experiments while isotonic crystalloid solution was used in others to create sufficient anemia to investigate the lower levels of oxygen delivery.

A given level of oxygen delivery was maintained for at least 30 min unless hemodynamic instability necessitated returning to a higher level of delivery. Oxygen consumption was measured every $5 \mathrm{~min}$. Oxygen consumption measurements made at low levels of delivery were followed by oxygen consumption measurements at higher, more physiologic levels of delivery to allow recovery. During transpulmonary shunting the ventilator rate was increased to maintain the $p \mathrm{CO}_{2}$ at $35-45 \mathrm{~mm} \mathrm{Hg}$. At every level of oxygen delivery, pulse, blood pressure, arterial oxygen content, mixed venous oxygen content, arterial $p \mathrm{O}_{2}, p \mathrm{CO}_{2}$, and $p \mathrm{H}$ were measured. Typically $3 \mathrm{hr}$ were required for cannulation and stabilization of the animal, with data collected for 3 to $9 \mathrm{hr}$ thereafter.

Oxygen consumption measurement. A closed circuit spirometry system was used (Fig. 1). A volume ventilator was used to compress a slave bellows that was included within the closed circuit. Tidal volumes from this bellows were directed to the dog's endotracheal tube by one-way valves. The inhalation valve operated in synchrony with the ventilator. Exhaust gases passed through a calcium hydroxide scrubber to remove carbon dioxide and were then returned to the bellows. An in-line calibrated magnetic valve on the exhalation side of the circuit created positive expiratory pressure $\left(5 \mathrm{cmH}_{2} \mathrm{O}\right)$. A volumetric spirometer within the circuit replaced oxygen taken up by the lungs, directly measuring oxygen consumption. The fraction of inspired oxygen within the circuit was set at 0.30 to 0.50 .

The absence of leaks in the spirometry circuit was confirmed at the close of each experiment by ventilating the dog after death had occurred and observing no change in the volume of the circuit.

Extracorporeal circuit (Fig. 2). Perfusion catheters were secured by purse-string sutures in the right atrial appendage and left atrial appendage. The superior vena cava was cannulated for venous drainage via the azygous vein. Occlusion tapes were passed around the superior vena cava and extracorporeal circulation was begun with reinfusion in the right atrium. The inferior vena cava was then cannulated and this venous return added to the extracorporeal circuit. All systemic venous return was being 
Right Atrial Bypass to Control Cardiac Output

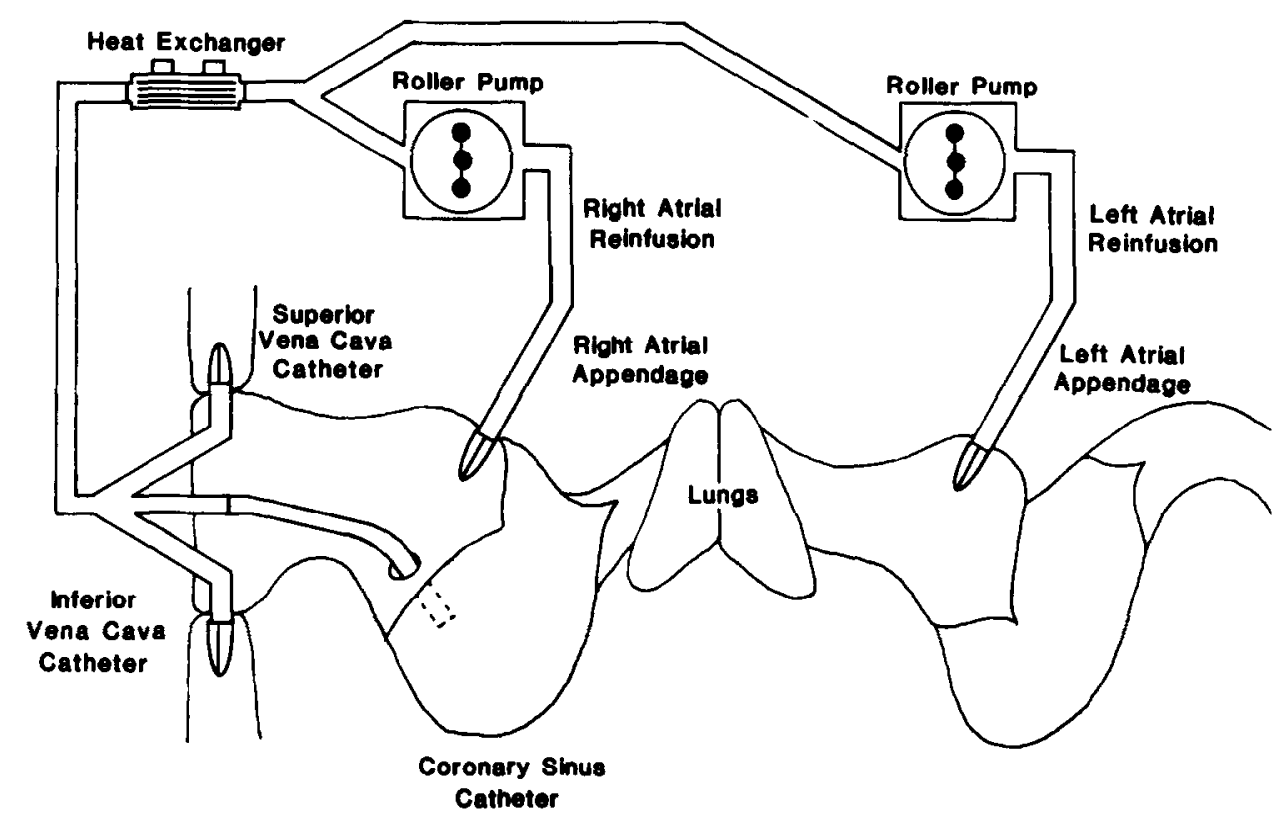

FIG. 2. Schematic diagram of right atrial bypass circuit used to mechanically control cardiac output. A second pump is a transpulmonary shunt to create hypoxia.

recirculated by the extracorporeal circuit. In six dogs a specially designed balloon-tipped catheter was introduced through the right atrium into the coronary sinus and this blood added to the venous drainage. The position of this catheter was confirmed by direct vision and palpation. Venous drainage (superior vena cava, inferior vena cava, coronary sinus) passed through a heat exchanger that maintained core temperature at $37^{\circ} \mathrm{C}$ and was then returned by a roller pump to the right atrial appendage. To create arterial hypoxia a second roller pump could be used to return a portion of this venous blood to the left atrium. A total of 3000 units of heparin sodium were added to the priming solution to provide anticoagulation.

Instrumentation and calculations. Oxyhemoglobin saturation was determined by an Instrumentation Laboratory model $282 \mathrm{Co}$-oximeter. This instrument measured the absorbance, specific for dog hemoglobin, at four wavelengths of light to determine the quantity of the various hemoglobin species present. The instrument then calculated the oxygen content using $1.39 \mathrm{cc}$ of oxygen per gram of hemoglobin as the oxygen carrying capacity for dog hemoglobin. The content of dissolved oxygen was then added by multiplying the $\mathrm{pO}_{2}$ by the solubility coefficient of oxygen in plasma at $37^{\circ} \mathrm{C}\left(0.0031 \mathrm{ccO}_{2} / \mathrm{mmHg}\right)$. The accuracy of this instrument when compared to standard reference methods has been confirmed in a recent report [7].

Atmospheric pressure was recorded using a mercury barometer on the day of each experiment. Oxygen consumption was measured at atmospheric pressure and temperature, saturated. These values were converted to standard temperature and pressure, dry, using standard conversion tables based upon the ideal gas behavior of atmospheric gases. The roller pumps were calibrated using volumetric collections of whole canine blood at different pump speeds. Oxygen delivery was calculated by multiplying the roller pump flow by the arterial oxygen content. All values are expressed as the mean \pm one standard deviation. Statistical significance was determined by the Student two-tailed $t$ test for comparing means. Correlation coefficients were determined by linear regression analysis.

All animals were cared for under guidelines established by the University of Michigan's Unit for Laboratory Animal Medicine.

\section{RESULTS}

Data from two animals (Nos. 6 and 10) are displayed in Table 1. Data are listed sequentially for each experiment. Note the return to baseline oxygen consumption after periods of low oxygen delivery. The pooled data for $\dot{D} \mathrm{O}_{2}$ and $\dot{V} \mathrm{O}_{2}$ from all dogs are plotted in Fig. 3. Absolute oxygen consumption and oxygen delivery values are given in $\mathrm{cc} / \mathrm{kg} / \mathrm{min}$. Eighty-one levels of oxygen delivery are represented. On the basis of stratified levels of oxygen delivery, sequential linear regressions were performed between oxygen delivery and oxygen consumption starting at the lowest levels of oxygen delivery increasing by increments of $1 \mathrm{cc} / \mathrm{kg} / \mathrm{min}$. The highest coefficient of cor- 


\section{TABLE 1}

Oxygen Delivery (cc/kg/min), Oxygen Consumption (cc/kg/min), Pumpflow (cc/kg/min), Hemoglobin (mg/dl), Partial Pressure of Oxygen in Arterial Blood (mmHg), Arterial Oxygen Content (cc/dl), and Venous Oxygen Content (cc/dl) during Two Experimental Sequences

\begin{tabular}{rccrrrr}
\hline$\dot{D O}_{2}$ & $\dot{V} \mathrm{O}_{2}$ & Pump CO & $\mathrm{Hb}$ & $P_{\mathrm{a}} \mathrm{O}_{2}$ & $C_{\mathrm{a}} \mathrm{O}_{2}$ & $C V \mathrm{O}_{2}$ \\
\hline 12.1 & 5.9 & 95 & 9.2 & 134 & 12.7 & 6.8 \\
8.2 & 5.7 & 85 & 8.8 & 48 & 9.7 & 3.3 \\
8.4 & 5.8 & 76 & 7.9 & 164 & 11.0 & 4.7 \\
5.9 & 4.8 & 80 & 8.0 & 42 & 7.3 & 2.3 \\
9.5 & 6.1 & 95 & 7.1 & 183 & 10.0 & 4.9 \\
4.3 & 3.8 & 81 & 7.0 & 36 & 5.3 & 1.4 \\
9.2 & 6.0 & 95 & 7.2 & 105 & 9.7 & 3.8 \\
1.8 & 1.6 & 40 & 6.5 & - & 4.4 & 0.8 \\
& & & & & & \\
13.1 & 6.3 & 68 & 13.6 & 330 & 19.4 & 10.9 \\
9.0 & 6.0 & 50 & 12.6 & 278 & 17.9 & 8.8 \\
12.7 & 6.1 & 76 & 12.2 & 187 & 16.7 & 10.1 \\
6.5 & 5.5 & 60 & 12.5 & 48 & 10.7 & 3.1 \\
11.4 & 6.1 & 76 & 10.9 & 175 & 15.0 & 8.2 \\
5.0 & 4.6 & 35 & 11.0 & 96 & 14.2 & 2.1 \\
11.7 & 5.9 & 76 & 11.2 & 160 & 15.4 & 7.9 \\
4.1 & 3.5 & 50 & 6.3 & 119 & 7.8 & - \\
\hline & & & & & &
\end{tabular}

relation between oxygen delivery and oxygen consumption occurred at oxygen delivery $\leqslant 8.0 \mathrm{cc} / \mathrm{kg} / \mathrm{min}$, corresponding to the inflection point in Fig. 3. For the 18 levels of oxygen delivery $\leqslant 8.0 \mathrm{cc} / \mathrm{kg} /$ min, oxygen consumption was linearly related to oxygen delivery $(r=0.96)$. The equation describing this linear relationship is $\dot{V} \mathrm{O}_{2}=0.69 \times \dot{D} \mathrm{O}_{2}$ +0.60 . The critical value of oxygen delivery, below which oxygen consumption was delivery dependent occurred at a coefficient of oxygen delivery of 1.4. Oxygen consumption was independent of oxygen delivery above an oxygen delivery of $8.0 \mathrm{cc} / \mathrm{kg} / \mathrm{min}(r=0.27)$. For the 63 levels of oxygen delivery above $8.0 \mathrm{cc} / \mathrm{kg} / \mathrm{min}$ the mean oxygen consumption was $5.8 \pm 0.5 \mathrm{cc} / \mathrm{kg} / \mathrm{min}$. The mean oxygen consumption for individual dogs varied from $4.5 \pm 0.3$ to $6.4 \pm 0.3 \mathrm{cc} / \mathrm{kg} / \mathrm{min}$ and remained stable over the course of each experiment. (Only 5 of 63 measurements of oxygen consumption differed from the mean oxygen consumption for a given dog by more than $10 \%$.) It should be noted that no attempt was made to normalize oxygen consumption to account for the variability of metabolic rates among the 11 dogs. Normalizing the oxygen consumption would simply decrease the variability seen in the deliveryindependent portion of the curve (i.e., oxygen delivery $>8.0 \mathrm{cc} / \mathrm{kg} / \mathrm{min}$ ) in Fig. 3 .

Mean blood pressure, measured $p \mathrm{H}$, and calculated bicarbonate concentration were also compared between high oxygen delivery states greater than $8.0 \mathrm{cc} / \mathrm{kg} / \mathrm{min}$ and low oxygen delivery states, less than $8.0 \mathrm{cc} / \mathrm{kg} / \mathrm{min}$. The mean blood pressure in the low delivery state was $80 \pm 32$ $\mathrm{mm}$ of mercury. The mean blood pressure in the higher delivery state was $110 \pm 26 \mathrm{~mm}$ of mercury $(P<0.05)$. The mean calculated bicarbonate concentrations in the low and high delivery states were $16.7 \pm 3.1$ and $18.2 \pm 2.4$ meq/liter, respectively $(P<0.05)$. The $p H$ 's were 7.20 \pm 0.087 and $7.26 \pm 0.081$ in the low and high delivery states, respectively $(P<0.05)$. The low oxygen delivery state was associated with hypotension and acidosis.

When the bypass pump flow (pump cardiac output) was compared to the calculated cardiac output based on the Fick relationship $\left(\mathrm{CO}=\dot{V} \mathrm{O}_{2} /\left(C_{\mathrm{a}} \mathrm{O}_{2}-C_{v} \mathrm{O}_{2}\right)\right)$ a correlation coefficient of 0.92 was obtained. The pump $\mathrm{CO}$ averaged $17 \%$ lower than the calculated CO.

\section{DISCUSSION}

The primary strengths of this model were the use of independent measurements to determine oxygen consumption and oxygen delivery and the mechanical control of oxygen delivery that it provided. In this acute animal model, oxygen delivery was manipulated over a wide range using right atrial bypass to mechanically control the cardiac output. Arterial oxygen content was varied by inducing hypoxia with a controlled right to left transpulmonary shunt and by hemodilutional anemia. Oxyhemoglobin saturation was measured by a co-oximeter of proven accuracy. Oxygen consumption was determined independently by direct measurement, using volumetric closed-circuit spirometry. These techniques avoid errors of mathematical coupling that may result when oxygen delivery and oxygen consumption are each calculated using the same measured variables [8]. Furthermore by mechanically controlling cardiac output there was no variation in cardiac output over time at a given pump speed and no drugs that may have undesirable effects on the metabolic rate were required to control cardiac output.

The use of extracorporeal circulation itself was a variable that could affect oxygen consumption. This method of bypass provided excellent control of cardiac output in a more physiologic fashion than other methods of mechanically controlling cardiac output, including right ventricular bypass or total cardiopulmonary bypass. It must be recognized that right atrial bypass as described in this experiment does not take into account bronchial

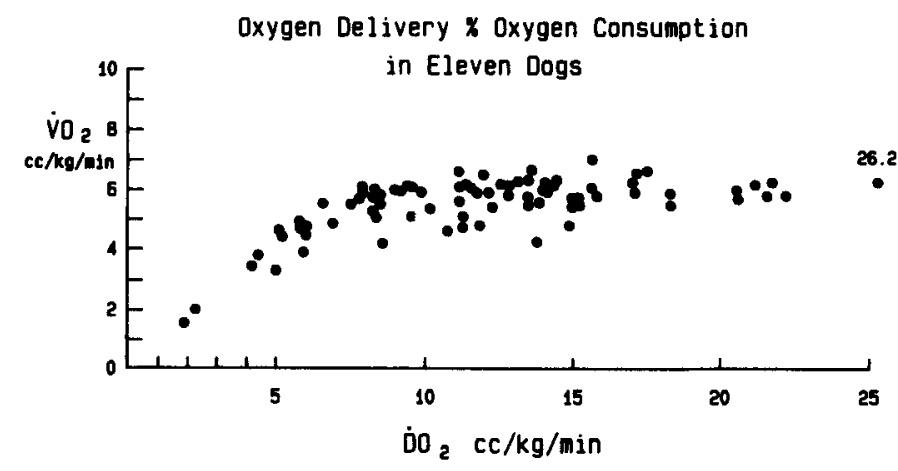

FIG. 3. Pooled data from 11 dogs. $V \mathrm{O}_{2}=0.69 \times D \mathrm{O}_{2}+0.60$ for $\mathrm{DO}_{2} \leqslant 8.0 \mathrm{cc} / \mathrm{kg} / \mathrm{min}$. 
blood flow or coronary blood flow that does not return by the coronary sinus. (This may represent up to $50 \%$ of coronary blood flow in dogs [9].)

Pancuronium paralysis does not raise oxygen consumption, but it may have an effect on oxygen consumption by preventing muscular activity or shivering that might play a role in normal variations in oxygen consumption. Likewise barbituate anesthesia may prevent physiologic responses that could affect the oxygen consumption. No attempt was made to take diurnal variations of oxygen consumption into account in this experiment. It should however be noted that baseline oxygen consumption remained very stable throughout the 3 - to $9-\mathrm{hr}$ period of measurement in each experiment.

In this experiment, anemia, hypoxia, and low cardiac output were used in combination to decrease oxygen delivery. Oxygen consumption was found to be independent of oxygen delivery above an oxygen delivery level of 8.0 $\mathrm{cc} / \mathrm{kg} / \mathrm{min}$. Below this level of oxygen delivery, oxygen consumption was a function of oxygen delivery. (As a point of reference, a dog with a hemoglobin of $14 \mathrm{~g} \%$ and a cardiac output of $120 \mathrm{cc} / \mathrm{kg} / \mathrm{min}$ has an oxygen delivery of approximately $23 \mathrm{cc} / \mathrm{kg} / \mathrm{min}$.) This low level of oxygen delivery $(8.0 \mathrm{cc} / \mathrm{kg} / \mathrm{min})$ represents the anaerobic threshold as indicated by the lower mean blood pressure, calculated bicarbonate, and $\mathrm{pH}$ levels.

Several studies in acute animal models also demonstrate that oxygen consumption is dependent upon oxygen delivery under the conditions of very low oxygen delivery. Adams, Dieleman, and Cain found a critical value of oxygen delivery in the rat of $23 \mathrm{cc} / \mathrm{kg} / \mathrm{min}$ [3]. Below this value oxygen consumption was linearly dependent upon oxygen delivery $\left(\dot{V} \mathrm{O}_{2}=0.78 \dot{D O}_{2}+0.89\right)$. They employed methods in which oxygen consumption was directly measured and oxygen delivery was calculated using the Fick relationship. Their data showed that the hypoxic, low oxygen delivery rats, were more acidotic $\left(p \mathrm{H} 7.24 p^{2} \mathrm{CO}_{2} 24\right.$ vs $p \mathrm{H} 7.41 \mathrm{CO}_{2} 40$ ) and had a lower mean arterial pressure than the normoxic rats. It was likely that anerobic metabolism was occurring and that this represented a deteriorating state. This critical value was well below the normal range of oxygen delivery in the rat $(50-70 \mathrm{cc} / \mathrm{kg} /$ $\mathrm{min}$ ) and corresponded to a coefficient of oxygen delivery of 1.2 .

Using both anemia and hypoxia to decrease the oxygen delivery, Cain found a critical level of oxygen delivery $(9.8 \mathrm{cc} / \mathrm{kg} / \mathrm{min})$ in dogs below which oxygen consumption was a function of oxygen delivery [4]. Again the animals were acidotic at low delivery levels indicating anerobic metabolism. Oxygen consumption and oxygen delivery were not derived independently.

Other investigators have found critical levels of oxygen delivery in other animal models as well. Oxygen consumption fell and acidosis developed in fetal lambs below fetal oxygen delivery rates of $0.6 \mathrm{mmole} / \mathrm{kg} / \mathrm{min}(13.4 \mathrm{cc} /$ $\mathrm{kg} / \mathrm{min}$ ) in one study and below $12-15 \mathrm{cc} / \mathrm{kg} / \mathrm{min}$ in another $[5,6]$.
Simmons et al. found that lactate rose in the serum of dogs subjected to low oxygen delivery states by either hypoxia or pulmonary artery occlusion [10]. Although they used estimated arterial oxygen content to determine oxygen delivery, they found that serum lactate rose at levels of oxygen delivery below $10 \mathrm{cc} / \mathrm{kg} / \mathrm{min}$.

In an anemic baboon model, Wilkerson $e t$ al. defined a detrimental level of anemia by an oxygen extraction ratio (ER) of $<50 \%$ [11]. This corresponds to a critical COD of 2.0 for the baboon. Oxygen consumption was constant and independent of delivery above that level.

In a recent report, Pepe and Culver independently determined oxygen consumption and oxygen delivery in a canine model [12]. They utilized expired gas analysis to calculate oxygen consumption. They manipulated cardiac output by changing the end expiratory pressure of the ventilator and by venous inflow occlusion. They calculated arterial oxygen content from blood gas data. In their groups of dogs, critically low levels of oxygen delivery were identified ( 11 to $14 \mathrm{cc} / \mathrm{kg} / \mathrm{min}$ oxygen delivery) below which oxygen consumption was linearly dependent upon delivery. This represents the only other study in which the variables of oxygen delivery and consumption were independently derived.

Clinical studies have demonstrated similar findings. Shibutani et al., using Fick calculated methods in anesthesized patients before the institution of cardiopulmonary bypass, found a critical value of oxygen delivery ( 330 $\mathrm{cc} / \mathrm{min} / \mathrm{m}^{2}$ or $8.2 \mathrm{cc} / \mathrm{min} / \mathrm{kg}$ ) below which oxygen consumption was linearly related to oxygen delivery [13]. Above this value oxygen consumption values plateaued. They also noted increased levels of arterial lactate at oxygen delivery below the critical value indicating anerobic metabolism. Chappell et al. found no change in oxygen consumption when oxygen delivery was increased by vasodilator therapy in patients with pulmonary hypertension or severe left ventricular failure [14]. These levels of oxygen delivery were above any critical level and supply dependency of oxygen consumption did not exist at the oxygen delivery levels measured in those disease states.

In contrast to the above studies that indicate supply dependency of oxygen consumption below a critically low level of oxygen delivery and supply independent consumption above this critical level, Mohsenifar et al. found that in patients with adult respiratory distress syndrome, oxygen consumption was linearly related to delivery up to very high levels of delivery $(21 \mathrm{cc} / \mathrm{kg} / \mathrm{min})$ [2]. Many of these patients were hypermetabolic. There is no data to indicate that these patients were either acidotic or unstable at the lower oxygen delivery states. Other clinical studies have reported supply dependency of oxygen consumption at high levels of oxygen delivery in certain disease states including the adult respiratory distress syndrome, hypovolemic and septic shock $[1,15]$. Plateau values of oxygen consumption were not found. These clinical studies have been criticized for the methods used to calculate oxygen consumption and oxygen delivery [8]. (Ox- 
ygen consumption was calculated by the Fick relationship, using the same measured variables used to calculate oxygen delivery.) This method guarantees mathematical coupling between consumption and delivery. Cain however has stated that the reported correlations between oxygen delivery and consumption are too high to be attributed solely to mathematical coupling [16]. Further clinical studies using independent measurement of oxygen delivery and oxygen consumption are needed to confirm these findings. When Annat et al. measured oxygen delivery and oxygen consumption independently in ARDS patients with normal blood lactate levels, $\dot{V} \mathrm{O}_{2}$ was independent of changes in $\dot{D} \mathrm{O}_{2}$ over a wide range of $\dot{D} \mathrm{O}_{2}$ [17]. This raises the possibility that previous clinical studies contain large elements of artifact.

Our report and the other animal studies cited above demonstrated supply dependent oxygen consumption only below a critical low level of oxygen delivery. Carefully controlled animal experiments using precise techniques such as those reported in the study are needed in septic animal models to further elucidate the relationship between oxygen delivery and utilization in sepsis.

This experiment demonstrated that throughout the normal physiologic range of oxygen delivery, oxygen consumption was independent of delivery in an acute canine model. It also showed that below critical levels of delivery (in this case a coefficient of oxygen delivery of 1.4 corresponding to an oxygen delivery of $8.0 \mathrm{cc} / \mathrm{kg} / \mathrm{min}$ ), oxygen consumption became supply dependent. Evidence from this experiment, as well as from others, indicates that anerobic metabolism occurred in these low oxygen delivery states. Our findings do not explain the supply dependent oxygen consumption that has been reported in the adult respiratory distress syndrome where supply is much greater than consumption and the coefficient of oxygen delivery is nearly normal $[4,5]$.

\section{REFERENCES}

1. Danek, S. J., Lynch, J. P., Weg, J. G., et al. The dependence of oxygen uptake on oxygen delivery in the adult respiratory distress syndrome. Amer. Rev. Respir. Dis. 122: 387, 1980.
2. Mohsenifar, Z., Goldbach, P., Tashkin, D. P., et al. Relationship between $\mathrm{O}_{2}$ delivery and $\mathrm{O}_{2}$ consumption in the adult respiratory distress syndrome. Chest 84(3): 267, 1983.

3. Adams, R. P., Dieleman, L. A., and Cain, S. M. A critical value for $\mathrm{O}_{2}$ transport in the rat. J. Appl. Physiol. 53(3): 660, 1982.

4. Cain, S. M. Oxygen delivery and uptake in dogs during anemic and hypoxic hypoxia. J. Appl. Physiol. 42(2): 228, 1977.

5. Wilkening, R. B., and Meschia, G. Fetal oxygen uptake, oxygenation, and acid-base balance as a function of uterine blood flow. Amer. J. Physiol. 244: H749, 1983.

6. Itskovitz, J., LaGamma, E. F., and Rudolph, A. The effect of reducing umbilical blood flow on fetal oxygenation. Amer. J. Obstet. Gynecol. 145: 813, 1983.

7. Dennis, R. C., and Valeri, C. R. Measuring percent oxygen saturation of hemoglobin, percent carboxyhemoglobin and methemoglobin, and concentrations of total hemoglobin and oxygen in blood of man, dog, and baboon. Clin. Chem. 26(9): 1304, 1980.

8. Archie, J. P. Relationship of oxygen delivery and oxygen consumption. Crit. Care Med. 12(8): 695, 1984.

9. Hammond, G. L., and Austen, W. G. Drainage patterns of coronary arterial flow as determined from the isolated heart. Amer. J. Physiol. 212(6): 1435, 1967.

10. Simmons, D. H., Alpas, A. P., Tashkin, D. P., et al. Hyperlactatemia due to arterial hypoxemia or reduced cardiac output, or both. $J$. Appl. Physiol. 45(2): 195, 1978.

11. Wilkerson, D. K., Rosen, A. L., Gould, S. A., Sehgal, L. R., Sehgal, H. L., and Moss, G. S. Oxygen extraction ratio: A valid indicator of myocardial metabolism in anemia. J. Surg. Res. 42: 629, 1987.

12. Pepe, P. E., and Culver, B. H. Independently measured oxygen consumption during reduction of oxygen delivery by positive endexpiratory pressure. Amer. Rev. Respir. Dis. 132: 788, 1985.

13. Shibutani, K., Komatsu, T., Kubal, K., et al. Critical level of oxygen delivery in anesthetized man. Crit. Care Med. 11(8): 640, 1983.

14. Chappell, T. R., Rubin, L. J., Markham, R. V., et al. Independence of oxygen consumption and systemic oxygen transport in patients with either stable pulmonary hypertension or refractory left ventricular failure. Amer. Rev. Respir. Dis. 128: 30, 1983.

15. Kaufman, B. S., Rackow, E. C., and Falk, J. L. The relationship between oxygen delivery and consumption during fluid resuscitation of hypovolemic and septic shock. Chest 85(3): 336, 1984.

16. Cain, S. R. Review: Supply dependency of oxygen uptake in ARDS: Myth or reality. Amer. J. Med. Sci. 288(3): 119, 1984.

17. Annat, G., Viale, J. P., Percival, C., Froment, M., and Motin, J. Oxygen delivery and uptake in the adult respiratory distress syndrome. Amer. Rev. Respir. Dis. 133: 999, 1986. 